\title{
Custos com Internações por Condições Sensíveis à Atenção Primária: séries temporais 2008-2015
}

\author{
Hospitalization costs for Ambulatory Care Sensitive Conditions: time Series 2008-2015
}

\author{
Como citar este artigo: \\ Sousa MEF, Sousa EC, Melo GAA, Carvalho REFL, Silva MRF, Pereira FGF. Hospitalization costs for Ambulatory Care Sensitive Conditions: time \\ Series 2008-2015. Rev Rene. 2020;21:e42091. DOI: https://doi.org/10.15253/2175-6783.20202142091
}

(D) Maria Evely Ferreira de Sousa ${ }^{1}$
(D) Eduardo Carvalho de Sousa
(D) Geórgia Alcântara Alencar Melo ${ }^{3}$
(D) Rhanna Emanuela Fontenelle Lima de Carvalho ${ }^{2}$
(D) Maria Rocineide Ferreira da Silva ${ }^{2}$
(D) Francisco Gilberto Fernandes Pereira ${ }^{1}$

${ }^{1}$ Universidade Federal do Piauí. Picos, PI, Brasil.

${ }^{2}$ Universidade Estadual do Ceará. Fortaleza, CE, Brasil.

${ }^{3}$ Universidade Federal do Ceará.

Fortaleza, CE, Brasil.

\section{Autor correspondente:}

Francisco Gilberto Fernandes Pereira

Rua Cicero Eduardo, 956. Junco.

CEP: 64607-670. Picos, PI, Brasil.

E-mail: gilberto.fp@hotmail.com

\section{RESUMO}

Objetivo: avaliar os custos com Internações por Condições Sensíveis à Atenção Primária, ponderando as séries temporais de 2008-2015. Métodos: estudo ecológico que se utilizou dos registros do Sistema de Informação Hospitalar. Amostra composta pelos registros de internações de diagnósticos incluídos na Lista Brasileira de Internações por Condições Sensíveis à Atenção Primária. Regressões polinomiais foram utilizadas para análise da tendência temporal das taxas ao longo do período. Resultados: houve decréscimo nas taxas por 10.000 habitantes (67.38 a 51.99) $\left(r^{2}=0,9783 ; p<0,001\right)$. As causas mais onerosas foram: asma, insuficiência cardíaca, cistite e pneumonia. Evidenciou-se tendência de redução das internações $(\beta=-2,15 ; p<0,001)$ e dispêndios médios $(\beta=-2243,24 ; p=0,169)$. Conclusão: $o$ diagnóstico asma obteve maior consumo, enquanto que a doença inflamatória do colo do útero atingiu menor quantitativo. Na série temporal, observou-se tendência de redução de gastos evidenciada pela diferença entre o maior custo no ano de 2009 e o menor, em 2015.

Descritores: Atenção Primária à Saúde; Serviços Básicos de Saúde; Qualidade, Acesso e Avaliação da Assistência à Saúde; Sistemas de Informação Hospitalar; Estudos Ecológicos.

\footnotetext{
ABSTRACT

Objective: to evaluate the costs of hospitalizations for ambulatory care sensitive conditions in the 2008-2015 time series. Methods: ecological study with records of the Hospital Information System. The sample was composed of records of admissions for diagnoses of the Brazilian List hospitalizations for ambulatory care sensitive conditions. Polynomial regressions were used to analyze the temporal trend of rates. Results: there was a decrease in the rates per 10,000 inhabitants (67.38 to 51.99) $\left(\mathrm{r}^{2}=0.9783\right.$; $\left.\mathrm{p}<0.001\right)$. The most costly causes were asthma, heart failure, cystitis, and pneumonia. There was a decreasing trend in hospitalizations $(\beta=-2.15 ; p<0.001)$ and expenditures $(\beta=-2243.24$; $\mathrm{p}=0.169$ ). Conclusion: the diagnosis asthma caused greater expenses, while inflammatory disease of the cervix reached caused lower expenses. In the time series, there was a trend towards reduction of expenses evidenced by the difference between the highest cost in 2009 and the lowest in 2015 .

Descriptors: Primary Health Care; Basic Health Services; Health Care Quality, Access, and Evaluation; Hospital Information Systems; Ecological Studies.
} 


\section{Introdução}

O Sistema Único de Saúde desde o surgimento perpassa por profundas transformações, marcadas por avanços, mas com importantes obstáculos a serem superados, como a qualidade dos serviços e assistência prestada à população, em todos os de níveis de complexidade: atenção primária, secundária, terciária e, atualmente, quaternária ${ }^{(1)}$.

No intuito de operacionalizar a organização desses níveis dentro do sistema de saúde, são mensurados indicadores da qualidade dos atendimentos e do acesso de usuários, de modo a garantir os princípios da equidade e da integralidade ${ }^{(2)}$. No entanto, devido às dificuldades geográficas, de recursos humanos, orçamento e infraestrutura do próprio sistema, os níveis secundário e terciário recebem demandas decorrentes de agravamentos de problemas de saúde que não são resolvidos na atenção primária ${ }^{(3)}$.

A Atenção Primária é a porta de entrada da população aos serviços de saúde, atuando na promoção e proteção da saúde, mostrando importância dentro da saúde pública, uma vez que orienta o sistema, de modo a contribuir para diminuição das superlotações em hospitais. Assim, torna-se categoria assistencial qualificada para beneficiar os indicadores de saúde da sociedade $^{(1)}$.

Nesse contexto, o Ministério da Saúde definiu as condições sensíveis à atenção primária como agrupamento de questões de saúde para os quais as adequadas intervenções da atenção primária tem como função minimizar o risco de internações hospitalares. Ações como prevenção, diagnóstico e tratamento precoce de doenças agudas; bem como controle e acompanhamento de doenças crônicas, devem ter como resultados a redução das Internações por Condições Sensíveis à Atenção Primária (ICSAP) ${ }^{(4-5)}$.

Elevados índices de internação por essas circunstâncias, em amostra populacional, resultam da dificuldade de acesso aos serviços de saúde, carência de dedicação adequada e satisfatória às questões de saúde, baixa vinculação à atenção primária, busca espontânea pelo atendimento especializado ou de urgência, ou pouca remediabilidade das condutas dos serviços. Assim, o crescente número de internações hospitalares requer vigilância e ampla investigação das causas para esse desfecho ${ }^{(6)}$.

Logo, verifica-se sistema público de saúde que oferece o serviço de Atenção Primária com fragilidades na forma de acesso aos usuários, com tendência para baixos indicadores de qualidade e resolubilidade, gerando, deste modo, hospitalizações desnecessárias; ao contrário, quando as ações deste nível são oferecidas de forma planejada e oportuna, as doenças crônicas e a gravidade decorrente delas tendem a decrescer e, consequentemente, evita a necessidade de hospitalizações ${ }^{(7)}$.

As ICSAP, indicadas como parâmetros de ingresso e qualidade, passaram a reconhecer a atenção primária à saúde, pois se tornou instrumento de verificação de desempenho, refletindo diretamente no planejamento orçamentário da saúde ${ }^{(8)}$. Ao partir desta premissa, investigações foram conduzidas acerca desta temática: no interior paulista, entre 2008 e 2010, as ICSAP oneraram $\mathrm{R} \$ 30.370 .691,08$, o que representou $17,0 \%$ dos investimentos totais em saúde ${ }^{(9)}$; em Santa Catarina, evidenciou-se diminuição no percentual dos gastos, entre 2001 e 2011, cujo coeficiente da regressão foi de 0,97 , valor que permite inferir redução de $3,0 \%$ ao ano na taxa de ICSAP, e de sete vezes, nos investimentos financeiros per capita em saúde ${ }^{(10)}$.

Ainda que o investimento destinado à saúde no Brasil em todos níveis de atenção seja escasso, observa-se disparidade em distribuição de recursos financeiros, principalmente quando se comparam os níveis de atenção à saúde entre si, pois há impressão revestida de viés que tende a compreender a Atenção Primária com menos complexidades e, portanto, menor necessidade de recursos tecnológicos, terapêuticos e diagnósticos ${ }^{(11)}$.

No cenário de realização, este estudo é inovador e com importância para saúde pública, uma vez que possibilitará a identificação dos custos pelas ICSAP. Deste modo, torna-se relevante, por viabilizar direcio- 
namento de ações para redução do total de dispêndios hospitalares, uma vez que ao serem analisados esses indicadores, será possível planejar estratégias que potencializem a redução dessas internações, bem como auxiliem gestores na verificação de áreas críticas do município. Logo, objetivou-se avaliar os custos com Internações por Condições Sensíveis à Atenção Primária, ponderando as séries temporais de 2008-2015.

\section{Métodos}

Realizou-se estudo ecológico, de tendência temporal, de janeiro a fevereiro de 2018, cuja unidade de análise foi o município de Picos, Piauí, Brasil, situado na Região Centro-Sul do Piauí, no Nordeste brasileiro, o qual possui estimativa populacional de 76.928 habitantes e área territorial de 577,304 km²(12).

No setor de saúde, Picos tem como órgão gestor a Secretaria Municipal de Saúde. Na Atenção Primária, as atividades são desenvolvidas por 36 Unidades Básicas de Saúde, correspondendo uma Estratégia Saúde da Família para cada unidade. A complementação da assistência à saúde no município é realizada através de Hospital de Médio Porte, que atende às demandas emergenciais de baixa e média complexidade, o qual tem verba advinda do município e do estado, e é administrado pela Fundação Piauiense de Serviços Hospitalares. Na macrorregião, Picos contabiliza cerca de 42 municípios, que também utilizam de serviços de saúde, por meio do sistema de referência e contrarreferência ${ }^{(13)}$.

A população do estudo foi composta pelos registros de hospitalizações ocorridas no referente município, nas quais o diagnóstico principal apontado no sistema de internação hospitalar, contido no Sistema Único de Saúde, tenha sido condição sensível à atenção primária, de acordo com a 10å Revisão da Classificação Internacional de Doenças (CID-10), nos anos de 2008 e 2015. Assim, a população foi analisada, visto que por se tratar de estudo ecológico, em que as anotações estão disponíveis na íntegra, analisar os dados em totalidade conferiu maior robustez às inferências.
As causas de internação e diagnósticos analisados estão descritos na Portaria 221, do Ministério da Saúde, de 17 de abril de 2008, presentes na 10 $0^{\text {a Classi- }}$ ficação Internacional de Doenças e Problemas Relacionados à Saúde: doenças preveníveis por imunização e condições sensíveis; gastrenterites infecciosas e complicações; anemia; deficiências nutricionais; infecções de ouvido, nariz e garganta; pneumonias bacterianas; asma; doenças das vias aéreas inferiores; hipertensão; angina; insuficiência cardíaca; doenças cerebrovasculares; diabetes mellitus; epilepsias; infecção nos rins e trato urinário; infecção da pele e tecido subcutâneo; doença inflamatória dos órgãos pélvicos femininos; úlcera gastrintestinal; doenças relacionadas ao pré-natal e parto; sífilis congênita; e síndrome da rubéola congênita ${ }^{(14)}$.

Os dados foram coletados por meio de informações secundárias acerca das Internações por Condições Sensíveis à Atenção Primária, originadas a partir das Autorizações de Internação Hospitalar contidas no banco informativo do Sistema de Informações Hospitalares, disponíveis de modo on-line, no site do Departamento de Informática do Sistema Único de Saúde (DataSUS $^{(15)}$. Adotou-se como série temporal o período entre 2008 e 2015, tendo em vista que, na ocasião do acesso, apenas estes anos estavam disponíveis.

A busca dos dados a serem inseridos na pesquisa foi orientada pelo fluxo: acesso à plataforma DataSUS ${ }^{(15)}$. Na aba de acesso à informação, primeiramente, escolheu-se o comando Informações de Saúde (TABNET); em seguida, Epidemiológicas e Morbidades; Morbidade Hospitalar do Sistema Único de Saúde/Sistema de Informação Hospitalar; Geral, por local de internação, a partir de 2008; depois, clicou-se no estado Piauí, no período de jan/2008 a dez/2015; Picos, e lista CID-10 - doença.

Os dados foram obtidos com a utilização da Lista Brasileira de Internações por Condições Sensíveis à Atenção Primária como instrumento de coleta de dados. A partir dessas informações, os dados foram alocados em planilha do Microsoft Excel e, após isto, as taxas foram calculadas utilizando-se como numerador 
o valor bruto das internações; e para o denominador, a população do período escolhido. Os dados foram normalizados para 10.000 habitantes, devido à população da cidade ser inferior a 100.000 moradores.

Utilizou-se do modelo de regressão polinomial para análise de tendência, considerando as causas das interações por condições sensíveis à atenção básica como variável dependente $(\mathrm{Y})$ e os anos como variável independente $(\mathrm{X})$. A tendência foi considerada significativa, quando o modelo estimado obteve $\mathrm{p}<0,05$.

Os resultados da regressão linear estão apresentados sob a forma do coeficiente $\beta$, nível de significância e o $\mathrm{R}^{2}$ ajustado. Quanto à autocorrelação serial, empregou-se o teste de Breusch Godfrey, admitindo-se autocorrelação, quando $\mathrm{p}<0,05$.

A pesquisa não envolveu qualquer tipo de intervenção (direta ou indireta) com seres humanos.
Foram utilizados elementos secundários, disponíveis em bancos de dados públicos, via on-line. Por isso, não necessitou de submissão à plataforma Brasil. No entanto, reafirma-se o compromisso ético de manipular os dados de acordo com originalidade destes, sem realizar alterações que atendessem às necessidades do pesquisador.

\section{Resultados}

A Tabela 1 mostra a distribuição das ICSAP em Picos, de modo geral, e segundo a doença, mostrando que no período estudado houve decréscimo nas taxas por 10.000 habitantes (67.38 a 51.99), comprovados mediante o coeficiente de determinação $\left(r^{2}=0,9783\right.$; $\mathrm{p}<0,001$ ).

Tabela 1 - Distribuição das taxas de Internações por Condições Sensíveis à Atenção Primária, segundo doença. Picos, PI, Brasil, 2018

\begin{tabular}{|c|c|c|c|c|c|c|c|c|c|c|}
\hline Doenças & 2008 & 2009 & 2010 & 2011 & 2012 & 2013 & 2014 & 2015 & $\mathbf{r}^{2 *}$ & $\mathbf{p}^{\dagger}$ \\
\hline Geral & 67,38 & 67,09 & 65,37 & 61,76 & 60,37 & 56,72 & 57,88 & 51,99 & 0,9783 & $<0,001$ \\
\hline Hepatite B & 0,27 & 0,41 & 0,13 & 0,13 & 0,39 & 0,92 & 0,65 & 0,26 & 0,9813 & 0,322 \\
\hline Febre Reumática & 0,27 & 0,54 & 0,13 & 0,26 & 0,13 & 0,13 & 0,39 & 0,26 & 0,5345 & 0,626 \\
\hline Malária & 0 & 0 & 0 & 0 & 0,13 & 0,13 & 0,13 & 0 & 0,9223 & 0,201 \\
\hline Anemia & 0,41 & 0,54 & 0,27 & 0,66 & 0,79 & 0,52 & 0,26 & 0,13 & 0,8258 & 0,427 \\
\hline Otite média & 2,48 & 2,19 & 2,58 & 2,26 & 1,85 & 1,44 & 1,31 & 0 & 0,9838 & 0,004 \\
\hline Faringite e Amigdalite & 0 & 0,68 & 0 & 0 & 0 & 0 & 0 & 0 & 0,6689 & 0,310 \\
\hline Asma & 175,77 & 241,30 & 195,33 & 189,41 & 146,39 & 143,60 & 126,45 & 93,80 & 0,9551 & 0,007 \\
\hline Bronquite & 4,69 & 9,31 & 6,94 & 12,93 & 14,70 & 17,09 & 12,58 & 10,71 & 0,9245 & 0,083 \\
\hline Enfisema/ Outras DPOC & 13,38 & 19,30 & 8,58 & 10,40 & 3,31 & 6,83 & 7,99 & 9,66 & 0,7789 & 0,130 \\
\hline Hipertensão & 38,08 & 58,47 & 57,61 & 50,42 & 42,65 & 30,64 & 12,58 & 11,10 & 0,9826 & 0,017 \\
\hline Insuficiência Cardíaca & 64,84 & 63,54 & 57,34 & 65,36 & 62,39 & 53,26 & 39,96 & 44,94 & 0,9849 & 0,011 \\
\hline Diabetes & 21,11 & 26,01 & 32,41 & 31,48 & 31,66 & 38,13 & 32,36 & 39,32 & 0,8897 & 0,005 \\
\hline Cistite & 25,80 & 39,85 & 50,26 & 42,81 & 44,38 & 55,49 & 50,32 & 47,55 & 0,9117 & 0,047 \\
\hline Úlcera gástrica duodenal & 0,96 & 0,82 & 0,95 & 0,80 & 1,19 & 1,05 & 0,13 & 0,26 & 0,9374 & 0,119 \\
\hline
\end{tabular}


As principais causas de ICSAP foram dos grupos diabetes mellitus $\left(r^{2}=0,8897 ; p=0,005\right)$, infecção no rim e trato urinário (cistite) $\left(\mathrm{r}^{2}=0,9117 ; \mathrm{p}=0,047\right)$ e doenças relacionadas ao pré-natal e parto (Sífilis Congênita) $\left(r^{2}=0,9832 ; p=0,015\right)$, permanecendo crescentes durante o período. Alguns grupos, como de infecções de ouvido, nariz e garganta (otite média e outros transtornos do ouvido médio após mastoidite) $\left(r^{2}=0,9838 ; p=0,004\right)$, asma $\left(r^{2}=0,9551 ; p=0,007\right)$, hipertensão (hipertensão essencial) $\left(\mathrm{r}^{2}=0,9826\right.$; $\mathrm{p}=0,017)$, insuficiência cardíaca $\left(\mathrm{r}^{2}=0,9849 ; \mathrm{p}=0,011\right)$ apresentaram proporção decrescente na maior parte do tempo. Os demais grupos permaneceram estáveis durante o tempo estudado, apresentando menor frequência de internação.

A Tabela 2 apresenta os dispêndios em $\mathrm{R} \$$ referentes às ICSAP, nos anos de 2008 a 2015, segundo a doença, as quais somaram valor de $\mathrm{R} \$ 13.166 .698,98$, sendo 2009 o ano de maior custo, totalizando $\mathrm{R} \$$ 1.988.211,38. Durante este período, a causa que totalizou ônus mais elevado foi asma, com R \$ 4.707.965,40; e menor gasto para doença inflamatória do colo do útero, com $\mathrm{R} \$ 342,84$.

A autocorrelação serial, realizada por meio do teste de Breusch-Godfrey, demonstrou valores de $\mathrm{p}=0,044$ para taxa de ICSAP e $\mathrm{p}=0,313$ para os gastos médios em reais. A partir disto, a análise de regressão polinomial foi realizada e evidenciou-se tendência de redução das ICSAP $(\beta=-2,15$; IC 95\%=-268 - -1,62; $\mathrm{p}<0,001 ; \mathrm{R}^{2}$ ajustado $\left.=0,933\right)$, e das despesas médias $\left(\beta=-2243,24 ;\right.$ IC $95 \%=-5757,5-1571,1 ; p=0,169 ; R^{2}$ ajustado $=0,170$ ) com as internações descritas, no período estudado.

Tabela 2 - Custo total (em R\$) com Internações por Condições Sensíveis à Atenção Primária, segundo doença. Picos-PI, Brasil, 2018

\begin{tabular}{|c|c|c|c|c|c|c|c|c|c|}
\hline Doenças & 2008 & 2009 & 2010 & 2011 & 2012 & 2013 & 2014 & 2015 & Total \\
\hline Coqueluche & 0 & 0 & 0 & 0 & 0 & 0 & 873 & 0 & 873 \\
\hline Hepatite B & 0 & 681 & 227 & 203 & 1.261 & 1.219 & 1.118 & 444 & 5.155 \\
\hline Febre Reumática & 403 & 832 & 187 & 406 & 243 & 187 & 610 & 428 & 3.300 \\
\hline Malária & 0 & 0 & 0 & 0 & 229 & 229 & 267 & 0 & 725 \\
\hline Anemia & 684 & 725 & 475 & 1.217 & 1.476 & 991 & 467 & 257 & 6.296 \\
\hline Otite média & 7.302 & 10.061 & 11.600 & 11.744 & 9.138 & 6.832 & 7.800 & 0 & 64.480 \\
\hline Faringite e amigdalite & 0 & 1527 & 0 & 0 & 0 & 0 & 0 & 0 & 1.527 \\
\hline Pneumonia & 219.044 & 297.518 & 186.690 & 245.117 & 173.582 & 285.210 & 361.965 & 327.268 & 2.096.397 \\
\hline Asma & 575.069 & 853.328 & 699.211 & 702.380 & 529.931 & 535.277 & 471.182 & 341.583 & 4.707 .965 \\
\hline Bronquite & 7.393 & 14.266 & 10.681 & 20.145 & 22.661 & 26.331 & 19.780 & 18.602 & 139.862 \\
\hline Enfisema & 46.611 & 73.070 & 32.352 & 42.529 & 12.926 & 27.561 & 26.944 & 34.045 & 296.040 \\
\hline Hipertensão & 50.842 & 82.105 & 85.706 & 72.389 & 64.624 & 44.098 & 18.231 & 16.420 & 434.417 \\
\hline Insuficiência Cardíaca & 307.310 & 334.744 & 303.074 & 362.125 & 344.544 & 295.983 & 223.065 & 253.549 & 2.424 .397 \\
\hline Diabetes & 48.011 & 72.809 & 87.824 & 92.836 & 96.585 & 111.814 & 95.248 & 114.196 & 719.326 \\
\hline Cistite & 216.175 & 237.198 & 279.825 & 298.219 & 359.000 & 288.546 & 259.469 & 250.534 & 2.188 .969 \\
\hline Salpingite e ooforite & 1.944 & 3.087 & 3.500 & 3.201 & 4.195 & 1.790 & 3.871 & 2.372 & 23.965 \\
\hline Doenças colo útero & 0 & 0 & 0 & 0 & 0 & 175 & 0 & 167 & 342 \\
\hline $\begin{array}{l}\text { Outras doenças órgãos pélvicos } \\
\text { femininos }\end{array}$ & 4.496 & 3.380 & 5.615 & 5.252 & 7.062 & 4.221 & 2.324 & 4.586 & 36.940 \\
\hline Úlcera gástrica/duodenal & 2.910 & 2.534 & 2.922 & 2.233 & 1.955 & 1.824 & 186 & 1.605 & 16.171 \\
\hline Sífilis Congênita & 0 & 338 & 258 & 258 & 517 & 1.035 & 517 & 2.646 & 5.572 \\
\hline Total & 1.488 .199 & 1.988 .211 & 1.710 .154 & 1.860 .261 & 1.629 .936 & 1.633 .330 & 1.495 .938 & 1.368 .709 & 13.166 .698 \\
\hline
\end{tabular}




\section{Discussão}

Por tratar de dados secundários, este estudo apresenta algumas limitações alusivas às anotações nos bancos de registros eletrônicos. Apesar do Sistema Único de Saúde possuir grande base de dados, os registros referem-se somente às hospitalizações efetivadas no âmbito deste sistema, as quais, embora majoritárias, não expressam a totalidade. Considera-se, ainda, o fato de que estes dados representam aspectos de área geograficamente limitada, o que inviabiliza a generalização para outras realidades.

A despeito das restrições desta pesquisa, os resultados poderão auxiliar gestores locais no planejamento, na implantação e avaliação de ações que se direcionem a aumentar a resolubilidade da atenção primária, com consequente redução de despesas em procedimentos de média e alta complexidade. Além disso, poderá colaborar com a efetivação da demanda dos fundamentos e das orientações do sistema único de saúde, entre estes, universalidade, integralidade e equidade.

As diversas transformações ocorridas no Sistema Único de Saúde têm instigado redução das ICSAP nas mais distintas localidades do Brasil ${ }^{(9)}$. Consequentemente, a atenuação dos dispêndios pode ser decorrente do fortalecimento da atenção primária, dado o incremento da cobertura da Estratégia Saúde da Família no país ${ }^{(16)}$, muito embora a natureza do tipo de estudo ecológico não seja suficiente para afirmar essa relação. Porém, enfatiza-se que, durante a década de 2000, a cidade de Picos elevou, quantitativamente, as equipes da Estratégia Saúde da Família, apresentando a maior cobertura populacional estimada do Piauí, Brasil. Atualmente, conta com 36 equipes desta Estratégia, cobrindo mais de 60 mil picoenses, em serviços de Atenção Primária à Saúde ${ }^{(13)}$.

Desse modo, considera-se que o processo de trabalho da Atenção Primária à Saúde pode ter relação com a ocorrência das ICSAP, compreendendo que é re- sultado, principalmente, da forma como se organizam os serviços de saúde, da formação e atuação dos profissionais de saúde, os quais podem tornar possível a redução nessas taxas de hospitalizações.

Entretanto, há outras variáveis que não foram levantadas nesta pesquisa, mas que podem ter relação direta ao indicador de ICSAP, como dados relativos às condições socioeconômicas da população e a forma de acesso aos serviços da Atenção Primária à Saúde, bem como o georreferenciamento da distribuição destes. Afirma-se, também, que a reestruturação no paradigma e na organização do modelo/sistema de saúde, com a expansão da Atenção Primária à Saúde e da Estratégia Saúde da Família, reorganizou e qualificou os serviços de saúde em Picos, mas não seria possível associar a queda de ICSAP somente a essa variável.Ao tecer paralelo a outros estudos, não se observou significância estatística na diminuição dos coeficientes de ICSAP e das despesas com atenção primária, em estudo realizado no Rio Grande do Sul, o qual apontou que os gastos em saúde, o ônus per capita e a cobertura da Estratégia Saúde da Família aumentaram significativamente, porém sem relação com as internações analisadas. Assim, inferiu-se que apesar dos investimentos financeiros e na cobertura populacional pela Estratégia Saúde da Família, ainda não foi suficiente para oferecer cuidados de saúde adequados à população ${ }^{(17)}$.

Em Rondônia, foi alta a frequência desse indicador entre 2012 e 2016 e, embora tenha havido incremento na cobertura da Estratégia Saúde da Família, no mesmo intervalo de tempo, esse acréscimo não foi significativo na frequência de ICSAP, para que mudanças ocorressem no estado $^{(18)}$.

No Paraná, estudo acerca do perfil de internamentos sensíveis à atenção primária em saúde constatou decréscimo entre 2000 e 2010, com redução de internações por doenças de elevada prevalência do Brasil, mas com manutenção de hospitalizações evitáveis por doenças cardiovasculares e respiratórias. 
Neste sentido, infere-se que serviços de atenção primária, com diretrizes e princípios bem delineados, reduzem o risco de agudização das condições crônicas e avalizam menores taxas de infecção por doenças transmissíveis ${ }^{(19)}$.

No tocante aos gastos, ao avaliar as taxas de hospitalizações evitáveis, em associação com a qualidade da atenção primária à saúde, identifica-se que os valores pagos por essas internações acompanham a queda de percentuais e taxas de $\operatorname{ICSAP}^{(20)}$.

Os custos constantemente elevados com o tratamento de condições relacionadas ao sistema respiratório, como asma, bronquite e pneumonia, podem ter relação com as características do clima local, que é seco, com temperatura média de $37^{\circ} \mathrm{C}$, e contaminado por partículas de poeira e fumaça, resultantes de queimadas, já que o referido município faz parte da região do semiárido piauiense ${ }^{(13)}$. Neste sentido, deve haver ação conjunta de políticas públicas que engajem intersetorialmente as esferas da gestão municipal, na busca por soluções de melhoria na qualidade do ar e ações permanentes de educação à população( $0^{(1)}$.

A economia dos dispêndios com essas internações evitáveis constitui real possibilidade de reverter tais recursos para aumentar a efetividade da atenção primária à saúde. Deste modo, é relevante que novas pesquisas que utilizem de métodos robustos de análise econômica e epidemiológica sejam realizadas, no sentindo de identificar o porquê do aumento real em valores com ICSAP, já que os recursos disponíveis na atenção primária e a própria expansão da Estratégia Saúde da Família parecem ser, teoricamente, suficientes para tratar essas doenças antes do surgimento de complicações ${ }^{(7,16)}$.

Enfatiza-se que a prevenção e o controle dessas doenças têm relação direta com o desempenho dos serviços de atenção primária. A magnitude da ocorrência desses tipos de internações aponta para necessidade de maior atenção à gestão da rede de atenção primária, no sentido de adequar a alocação dos recursos estratégicos e financeiros, de acordo com as demandas da população, as quais devem ser suscitadas a partir de diagnóstico situacional periódico. Além disso, é recomendável a racionalização e fiscalização para uso apropriado dos recursos disponíveis, de modo que haja maior eficiência no campo assistencial ${ }^{(9)}$.

A realização de melhorias na atenção primária à saúde, com investimentos estruturais e conforme as características essenciais, sejam a facilidade de acesso, a integralidade da atenção, a coordenação efetiva da atenção, a consideração do contexto familiar e o estabelecimento de vínculos entre os serviços e a população, são ações que podem contribuir com a diminuição da ocorrência das ICSAP no município ${ }^{(9)}$.

O papel da enfermagem nessa redução é importante, na medida em que o trabalho na atenção primária à saúde inclui assistência interdisciplinar em equipe para integralidade das ações, com educação permanente, nas quais priorizem o cuidado para promoção da saúde, prevenção e controle de riscos e agravos de doenças que integram a lista brasileira de $\operatorname{ICSAP}^{(20)}$.

\section{Conclusão}

O diagnóstico asma obteve maior custo, enquanto que a doença inflamatória do colo do útero apresentou menor. Na série temporal, observou-se tendência de redução de gastos evidenciada pela diferença entre o maior dispêndio no ano de 2009 e o menor, em 2015.

\section{Colaborações}

Sousa MEF, Sousa EC e Pereira FGF contribuíram com concepção e projeto ou análise e interpretação dos dados. Melo GAA colaboraram com redação do artigo e revisão crítica relevante do conteúdo intelectual. Carvalho REFL e Silva MRF cooperaram com a aprovação final da versão a ser publicada. 


\section{Referências}

1. Miranda GMD, Mendes ACG, Silva ALA. The challenge of organizing a universal and efficient National Health System in the Brazilian federal pact. Saúde Soc. 2017; 26(2):329-35. doi: https:// doi.org/10.1590/S0104-12902017168321

2. Barros FPC, Sousa MF. Equity: concepts, meanings and implications for the Brazilian National Health Sys Saúde Soc. 2016; 25(1):9-18. doi: https://doi. org/10.1590/S0104-12902016146195

3. Barbosa ML, Celino SDM, Carneiro AG, Costa GMC. Health care provided by SUS: the experience of foreign students. Esc Anna Nery 2016; 20(4):e20160092. doi: dx.doi.org/10.5935/14148145.20160092

4. Rehem TCMSB, Ciosak SI, Egry EY. Primary Care Sensitive Hospitalization: users detect flaws on the access to services. Rev Esc Enferm USP. 2014; 48(Esp2):70-6. doi: https://doi.org/10.1590/ S0080-623420140000800011

5. Santos LM, Gonçalves MA, Charles C. As despesas municipais em saúde impactam as Internações por Condições Sensíveis à Atenção Primária (ICSAP)? Uma análise em municípios de Minas Gerais. Rev Gestão Sistema Saúde. 2016; 5(1):6275. doi: https://doi.org/10.5585/rgss.v5i1.185

6. Avelino CCV, Goyatá SLT, Nogueira DA, Rodrigues LBB, Siqueira SMS. Quality of primary health care: an analysis of avoidable hospitalizations in a Minas Gerais county, Brazil. Ciênc Saúde Coletiva. 2015; 20(4):1285-93. doi: http://dx.doi. org/10.1590/1413-81232015204.12382014.

7. Costa LQ, Pinto Júnior EP, Silva MGC. Time trends in hospitalizations for Ambulatory Care Sensitive Conditions among children under five years old in Ceará, Brazil, 2000-2012. Epidemiol Serv Saúde. 2017; 26(1):51-60. doi: https://doi.org/10.5123/ S1679-49742017000100006

8. Arruda JS, Dias da Costa JS. Hospitalizations for primary care sensitive conditions in Novo Hamburgo, Rio Grande do Sul. Rev Bras Med Fam Comunidade. 2017; 12(39):1-11. doi: http:// dx.doi.org/10.5712/rbmfc12(39)1256
9. Ferreira JBB, Borges MJG, Santos LL, Forster AC. Internações por condições sensíveis à atenção primária à saúde em uma região de saúde paulista, 2008 a 2010. Epidemiol Serv Saúde. 2014; 23(1):45-56. doi: http://dx.doi.org/10.5123/ S1679-49742014000100005

10. Brasil VP, Costa JSD. Hospitalizations owing to ambulatory care sensitive conditions in Florianopolis, Santa Catarina, Brazil - an ecological study, 2001-2011. Epidemiol Serv Saúde. 2016; 25(1):75-84. doi: http://dx.doi.org/10.5123/ S1679-49742016000100008

11. Harrison MJ, Dusheiko M, Sutton M, Gravelle H, Doran T, Roland M. Effect of a national primary care pay for performance scheme on emergency hospital admissions for ambulatory care sensitive conditions: controlled longitudinal study. BMJ. 2014; 349:6423. doi: https://doi.org/10.1136/ bmj.g6423

12. Instituto Brasileiro de Geografia e Estatística (IBGE). Indicadores de Base Populacional [Internet]. 2019 [citado 2019 Ago 16]. Disponível em:http://cidades.ibge.gov.br/xtras/perfil. php? codmun $=220800$

13. Prefeitura Municipal de Picos. Secretaria Municipal de Saúde de Picos. Plano Diretor do Município de Picos [Internet]. 2019 [citado 2019 ago 16]. Disponível em: http://www2.picos.pi.gov.br/\#!

14. Ministério da Saúde (MS). Secretaria de Atenção à Saúde. Portaria no 221, de 17 de abril de 2008. Define a lista brasileira de Internações por Condições Sensíveis à Atenção Primária. [Internet]. 2008 [citado 2019 Aug 16]. Disponível em: http://bvsms.saude.gov.br/bvs/saudelegis/ sas/2008/prt0221_17_04_2008.htm

15. Ministério da Saúde (BR). DATASUS. Dados de morbidade hospitalar do SUS (SIH/SUS) [Internet]. 2015 [citado 2019 Nov 01]. Disponível em: http://tabnet.datasus.gov.br/cgi/deftohtm.exe?sih/cnv/nipi.def

16. Souza QK, Peixoto SV. Descriptive study on the evolution of hospitalization costs for ambulatory care sensitive conditions in Brazil, 20002013. Epidemiol Serv Saúde. 2017; 26(2):28594.doi:http://dx.doi.org/10.5123/S167949742017000200006 
17. Soranz D, Pinto LF, Penna GO. Themes and Reform of Primary Health Care (RCAPS) in the city of Rio de Janeiro, Brazil. Ciênc Saúde Coletiva. 2016; 21(5):1327-38. doi: http://dx.doi. org/10.1590/1413-81232015215.01022016

18. Santos BV, Lima DS, Fontes CJF. Hospitalization for ambulatory care-sensitive conditions in the state of Rondônia, Brazil: a descriptive study of the period 2012-2016. Epidemiol Serv Saúde. 2019; 28(1):e2017497. doi: http://dx.doi.org/10.5123/ S1679-49742019000100001
19. Borges PKO, Schawb PM, Blanski CR, Floriano LSM, Lopes BG, Muller EV. Sensitive hospitalizations to primary care and care in the health care network. Rev Rene. 2016; 17(5):668-75. doi: http://dx.doi. org/10.15253/2175-6783.2016000500012

20. Conceição RS, Silveira GSP, Veiga AJP, Matta JMB. Air temperature and its relationship with some respiratory diseases in Victory Conquest - BA. Rev Geoaraguaia [Internet]. 2015 [cited Nov 1, 2019]; 5(2):69-81. Available from: http:// periodicoscientificos.ufmt.br/ojs/index.php/ geo/article/view/4929/pdf_28

\section{(cc) BY}

Este é um artigo de acesso aberto distribuído sob os termos da Licença Creative Commons 